\title{
Protocol to disseminate a hospital-site controlled intervention using audit and feedback to implement guidelines concerning inappropriate treatment of asymptomatic bacteriuria
}

Barbara W. Trautner ${ }^{1,12^{*}}$, Pooja Prasad ${ }^{2}$, Larissa Grigoryan ${ }^{3}$, Sylvia J. Hysong ${ }^{1,12}$, Jennifer R. Kramer ${ }^{1,12}$, Suja Rajan ${ }^{4}$, Nancy J. Petersen ${ }^{1,12}$, Tracey Rosen ${ }^{1,12}$, Dimitri M. Drekonja ${ }^{5}$, Christopher Graber ${ }^{6}$, Payal Patel ${ }^{7}$, Paola Lichtenberger ${ }^{8}$, Timothy P. Gauthier ${ }^{8}$, Steve Wiseman ${ }^{7}$, Makoto Jones ${ }^{9}$, Anne Sales ${ }^{10}$, Sarah Krein ${ }^{11}$, Aanand Dinkar Naik ${ }^{1,12}$ and The Less is More Study Group

\begin{abstract}
Background: Antimicrobial stewardship to combat the spread of antibiotic-resistant bacteria has become a national priority. This project focuses on reducing inappropriate use of antimicrobials for asymptomatic bacteriuria (ASB), a very common condition that leads to antimicrobial overuse in acute and long-term care. We previously conducted a successful intervention, entitled "Kicking Catheter Associated Urinary Tract Infection (CAUTI): the No Knee-Jerk Antibiotics Campaign," to decrease guideline-discordant ordering of urine cultures and antibiotics for ASB. The current objective is to facilitate implementation of a scalable version of the Kicking CAUTI campaign across four geographically diverse Veterans Health Administration facilities while assessing what aspects of an antimicrobial stewardship intervention are essential to success and sustainability.

Methods: This project uses an interrupted time series design with four control sites. The two main intervention tools are (1) an evidence-based algorithm that distills the guidelines into a streamlined clinical pathway and (2) casebased audit and feedback to train clinicians to use the algorithm. Our conceptual framework for the development and implementation of this intervention draws on May's General Theory of Implementation. The intervention is directed at providers in acute and long-term care, and the goal is to reduce inappropriate screening for and treatment of ASB in all patients and residents, not just those with urinary catheters. The start-up for each facility consists of centrally-led phone calls with local site champions and baseline surveys. Case-based audit and feedback will begin at a given site after the start-up period and continue for 12 months, followed by a sustainability assessment. In addition to the clinical outcomes, we will explore the relationship between the dose of the intervention and clinical outcomes.

(Continued on next page)
\end{abstract}

\footnotetext{
* Correspondence: trautner@bcm.edu

${ }^{1}$ Center for Innovations in Quality, Effectiveness, and Safety (IQuESt) (152),

Michael E. DeBakey Veterans Affairs Medical Center, 2002 Holcombe

Boulevard, Houston, TX 77030, USA

${ }^{12}$ Baylor College of Medicine in Houston, Houston, TX, USA

Full list of author information is available at the end of the article
} 
(Continued from previous page)

Discussion: This project moves from a proof-of-concept effectiveness study to implementation involving significantly more sites, and uses the General Theory of Implementation to embed the intervention into normal processes of care with usual care providers. Aspects of implementation that will be explored include dissemination, internal and external facilitation, and organizational partnerships. "Less is More" is the natural next step from our prior successful Kicking CAUTI intervention, and has the potential to improve patient care while advancing the science of implementation.

Keywords: Antibiotic stewardship, Asymptomatic bacteriuria, Audit and feedback, Guidelines implementation, Dissemination, Urinary tract infection

\section{Background}

One of the most common reasons for overuse of antibiotics in both acute and long-term care is inappropriate treatment of asymptomatic bacteriuria (ASB), or bacteria in the urinary tract without related urinary symptoms [1-3]. Unnecessary antibiotics given to treat ASB can cause harm in terms of antibiotic resistance, adverse drug effects, and needless expense [4]. The need in the Veterans Health Administration (VHA) to improve this practice is particularly acute, as the VA Antimicrobial Stewardship Task Force reported in April 2016 that 72\% of cases of ASB were treated unnecessarily with antibiotics [5].

We designed and validated a successful antimicrobial stewardship intervention to decrease guidelinediscordant management of ASB in Veterans in hospitals and long-term care [6]. This "Kicking CAUTI Campaign" led to a $71 \%$ reduction in screening for ASB and a $75 \%$ reduction in treatment of ASB at one major VA medical center [7]. The current dissemination project, entitled "Less is More," will evaluate the effectiveness of the Kicking CAUTI Campaign across four different VHA medical centers while assessing the adoption, fidelity, generalizability, and necessary dose of the intervention (Fig. 1). We will also measure effectiveness of the program with respect to reducing inappropriate screening for and treatment of ASB. Unlike the original Kicking CAUTI intervention, we will include all cases of bacteriuria, occurring with or without a urinary catheter, to be more broadly applicable, as UTI is a much more common reason for antibiotic use than CAUTI.

The Kicking CAUTI Campaign was controlled, grounded in evidence, and employed audit and feedback as part of a multifaceted strategy in acute and long-term care wards $[6,7]$. The two main tools used in this intervention are (1) an evidence-based, actionable ("fast and frugal") algorithm $[8,9]$ which distills the guidelines into a streamlined clinical pathway and encourages a mindful pause and (2) case-based audit and feedback to train clinicians to use the algorithm within context. The mindfulness engendered by the algorithm then becomes embedded into the mental rules of thumb (heuristics) clinicians use in routine care, thus correcting misleading cognitive biases [9-11]. We chose audit and feedback as part of our intervention, as it had been shown to be successful for reducing overuse of antibiotics in prior studies, [12-17] and it is one of the evidence-based strategies recommended by guidelines on antimicrobial stewardship [18].

Current evidence to support antibiotic stewardship interventions is limited. Although multiple studies suggest that antimicrobial stewardship programs may be associated with improved antibiotic prescribing practices, the quality of the evidence is low, generalizability is unclear, and cause and effect are unproven. For example, in 2016, the Infectious Diseases Society of America issued guidelines on "Implementing an Antibiotic Stewardship Program," and of the 28 recommendations made, 23 are supported by weak evidence or expert opinion [19]. The Kicking CAUTI intervention represents an advance beyond prior stewardship studies while addressing important knowledge gaps.

The Less is More project will provide generalizable information for the field of implementation science because a theoretical model guides both the work and the assessment processes. Each aspect of our intervention is linked to a critical concept in the General Theory of Implementation (Fig. 2) [20]. We will measure the "dose" delivered of various components of the intervention and relate the dose intensity to the clinical outcomes. We are also interested in assessing the context in which the intervention occurs, including both individual and institutional factors, using measures that will assist in pooling data across studies. Thus, this project will further contribute to our understanding of the use and utility of key implementation strategies, including external and internal facilitation [21,22] and audit and feedback, in both inpatient and long-term care settings.

\section{Methods and design \\ Study design}

The study uses interrupted time series design with four contemporary control sites to test an intervention using audit and feedback of healthcare providers to improve their compliance with ASB guidelines. The intervention will be conducted at four geographically diverse VA 


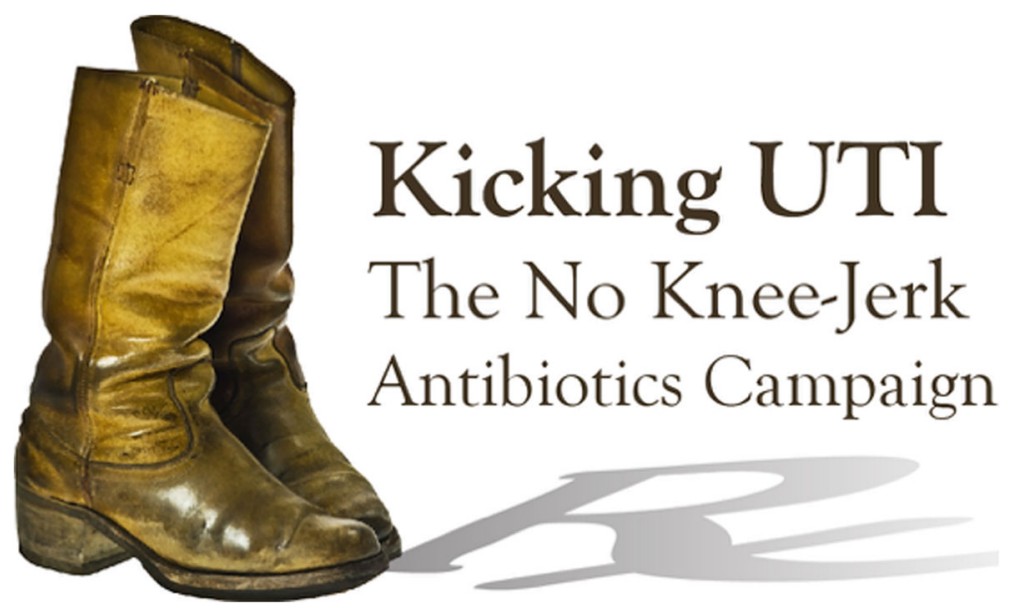

Fig. 1 Study logo for Less is More project

facilities, with external facilitation provided by the coordinating site in Houston. The control sites will be four VA facilities in the same geographic area as each intervention site to account for any potential regional differences in policies or practices regarding antibiotic stewardship.

\section{Specific aims and hypotheses}

Specific Aim 1, which will be performed in year 1, is to assess context, barriers, and facilitators at each site prior to implementation. This work includes baseline measurements of ASB diagnosis and treatment to inform intervention implementation.

Specific Aim 2, performed over years 1-3, is to evaluate implementation of a scalable version of the Kicking CAUTI intervention (individualized, case-based audit and feedback) in four geographically distinct VA medical centers, including both acute and long-term care settings, with four contemporaneous controls. We hypothesize that the intervention observation periods will exhibit a decrease in screening urine cultures ordered (primary outcome), decreased use of antimicrobials, and a decreased number of episodes of Clostridium difficile infection, without an increase in urinary source bacteremia, among intervention sites compared to control sites (Aim 2A). We also hypothesize that higher levels of adoption and fidelity will be associated with better clinical outcomes (Aim 2B).

Specific Aim 3, performed throughout the 3 years of the project, is to assess the financial implications of the intervention through a budget impact analysis [23].

\section{Conceptual model}

Our conceptual framework for the development and implementation of a scalable version of the Kicking CAUTI intervention draws on May's proposed General Theory of Implementation, which links Normalization Process Theory (NPT), [20, 24] with other sociological and psychological theories (Fig. 2). The General Theory of

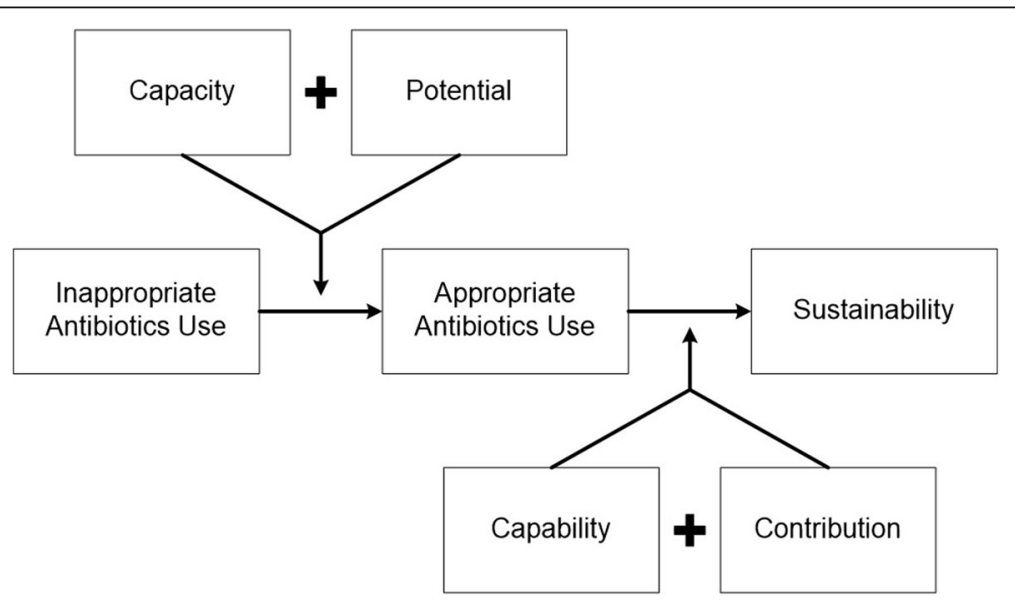

Fig. 2 Conceptual Model for the Less is More Intervention 
Implementation describes constructs that must be assessed and that serve as potential targets in the process of implementing a complex intervention successfully [20]. These include factors that improve the capacity of agents to cooperate and coordinate in order to implement (e.g., material resources, social roles and norms, and cognitive resources), as well as their potential (individual intention and collective commitment) for implementation. A complex intervention succeeds and becomes embedded in daily routines through the capability of individuals to operationalize a complex intervention. Finally, a sustainable intervention requires ongoing contribution by the parties involved. We expect that over time, the mindful pause prompted by our algorithm will become a standard heuristic through which guideline-concordant behaviors will be normalized into routine care. Our study measurements and variables are linked to our conceptual model (Table 1).

\section{Overview of intervention design and timeline}

Our overall objective in this proposal is to evaluate the effectiveness and implementation of a streamlined, scalable version of the Kicking CAUTI Campaign across four diverse VA medical centers. To address scalability, we will gather our primary clinical outcome data from the VA Corporate Data Warehouse (CDW), which is a relational database extracted directly from patients' electronic medical records containing all laboratory results, including cultures. We will train existing antimicrobial stewardship champions at each site (generally infectious diseases physicians and infectious diseases pharmacists) to deliver the intervention, thus using existing resources. The 3-year project allows time for three phases at each site: project startup (surveys and baseline data measurements), 12 months for active intervention implementation, and a sustainability phase during which active support from the centralized coordinating site ceases (Fig. 3). Audit and feedback will take place on two levels for this project. Provider level audit and feedback will consist of case based training in applying the ASB guidelines to individual patients. The local champions will deliver this in small group settings. Facility level audit and feedback will consist of information on urine cultures ordered and antibiotic use on a monthly basis, compared to other participating facilities (intervention and comparison). The facility level feedback will be given to each site champion for distribution to relevant parties within their site.

\section{Study setting}

We will conduct this study in inpatient acute medicine units and long-term care units in the following four major VA medical centers: Ann Arbor, Greater Los Angeles, Miami, and Minneapolis. Each of these sites has both acute care and community living centers. These sites were chosen because each has a motivated local physician champion who is early to mid-career and has the bandwidth to commit to this project, with support from a senior antimicrobial stewardship mentor on site. The proposed comparison sites are Madison, San Francisco, Tampa, and Milwaukee. The four comparison facilities have been chosen from the same geographic areas as the intervention sites and matched in terms of teaching status, characteristics of their current antimicrobial stewardship program, ward types, facility level, ICU level, and number of beds.

\section{Participants}

The goal of this project is to teach providers to distinguish between UTI and ASB and to make the appropriate choice to withhold antimicrobials when the patient has ASB. Thus, the health care providers who order urine cultures and antimicrobial agents are the subjects of the intervention itself, and their involvement will be to receive education about the relevant guidelines and audit and feedback about their management of bacteriuria, both catheter-associated and not catheterassociated. The clinical outcomes for this project, such as number of urine cultures ordered and antibiotic use for bacteriuria, will be monitored in the Veterans who are inpatients in acute care facilities or residents of longterm care facilities within intervention and control sites. The local project team at each implementation site consists of a site champion (an infectious diseases physician), an infectious diseases pharmacist, and a research assistant, plus any other members recruited by the site champion.

\section{Materials \\ Surveys}

We will administer three different surveys in year 1, each for a different purpose. These include the site elements questionnaire, [25] the context scale of the Organizational Readiness to Change Assessment (ORCA) instrument, [26] and the Kicking UTI survey [27]. The ORCA was initially developed through the Ischemic Heart Disease Quality Enhancement Research Initiative (QUERI) program and has been used in several QUERI related studies [26, 28, 29]. We plan to administer the ORCA context assessment, which asks about leadership culture, staff culture, leadership practice, and evaluation/accountability. The main purpose of the Kicking CAUTI survey is to use this previously validated tool to assess baseline awareness of the CAUTI and ASB guidelines and to identify the cognitive biases driving overtreatment of ASB, which will enable us to target our intervention teaching materials. The Kicking UTI survey will be administered widely to licensed providers who 
Trautner et al. Implementation Science (2018) 13:16

Page 5 of 11

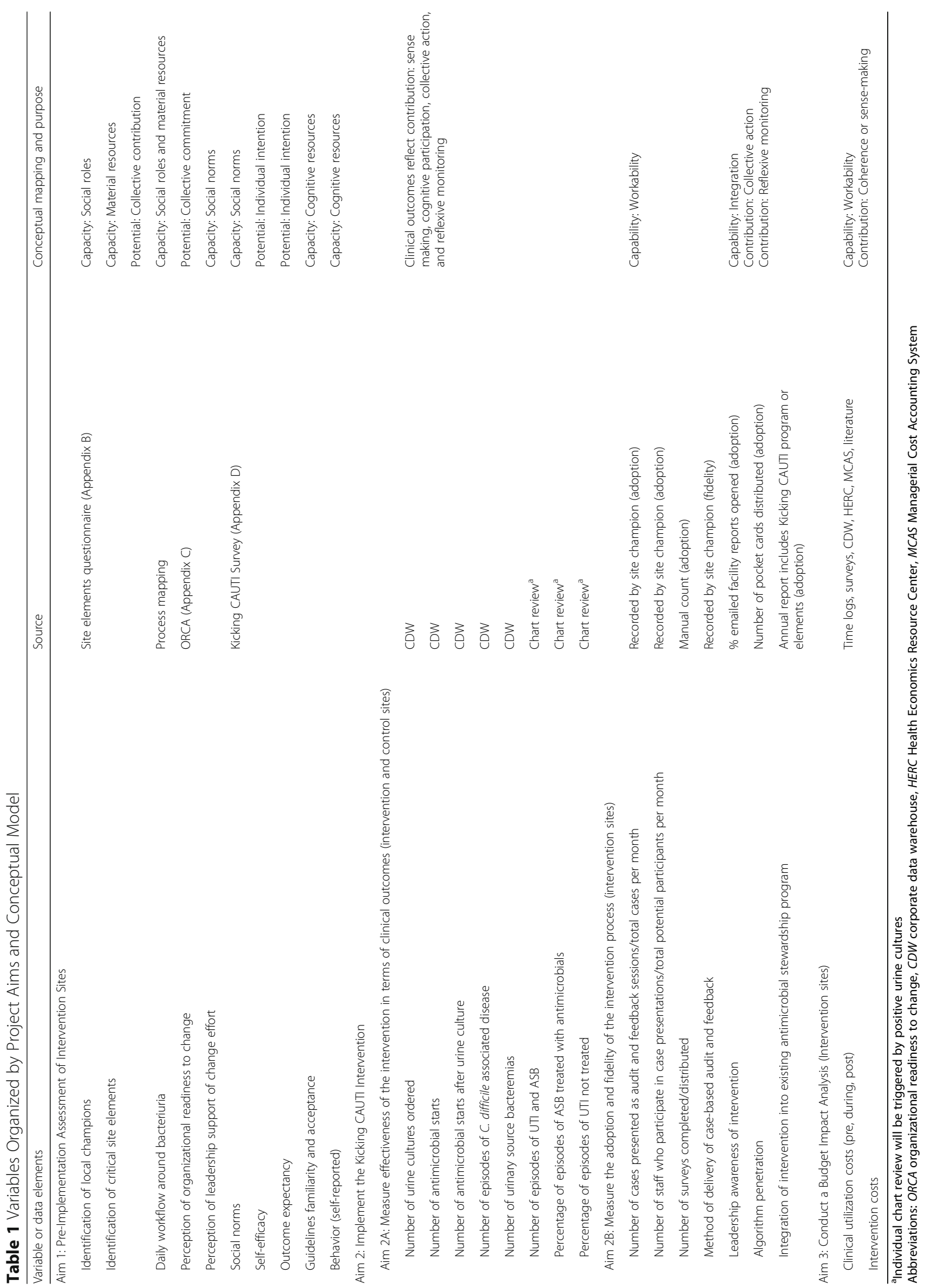




\section{Project Timeline for Less is More Campaign}

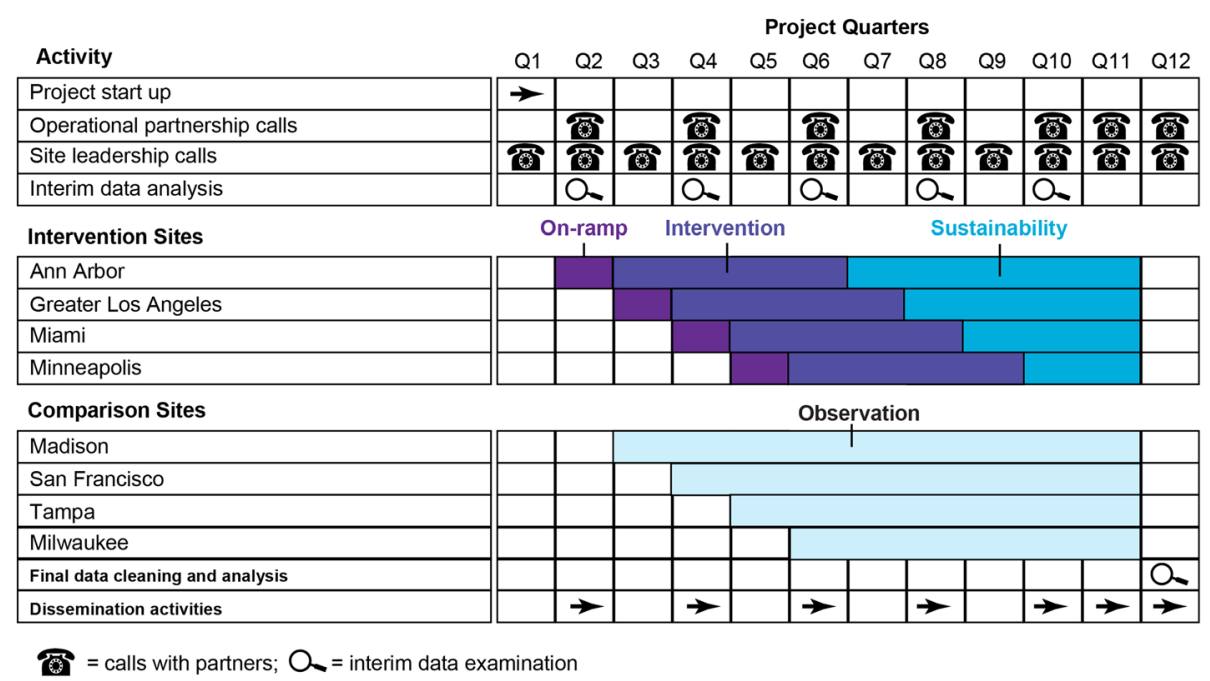

Fig. 3 Project Timeline for Roll-out of the Less is More Intervention

order antibiotics, nurses, and clinical nurse assistants. The site elements questionnaire [25] will ask questions about available resources, such as whether any antimicrobial restriction policies are in place, whether the site has an infectious diseases physician, etc. A single site elements survey will be completed per site, by the site champion, about local resources available for antibiotic stewardship.

\section{Algorithm and teaching materials}

The main tool used in our intervention is our evidencebased, actionable ('fast and frugal') Kicking UTI algorithm $[8,9]$. This algorithm distills the guidelines into a streamlined clinical pathway and encourages a mindful pause. Specifically, the algorithm applies to any patient who is being assessed for possible UTI. The provider using the algorithm is instructed to ask themselves two questions before reflexively ordering a urine culture or starting empiric antibiotics. These questions are: "Does the patient have any of these evidence-based symptoms of UTI," and "If yes, then could a non-urinary condition account for these symptoms?" During the intervention, the project team will create teaching materials based on specific cases, each built around this algorithm. Delivery of these case-based teaching materials to the providers involved in the case constitutes our audit and feedback. Each case is presented in a brief PowerPoint which begins with the algorithm, hyperlinked at each decision point. The provider receiving the feedback can thus explore possible choices and options during the brief educational session delivered by a member of the local project team (physician, pharmacist, or research assistant).

\section{Intervention}

Our intervention uses case-based audit and feedback to train clinicians to use the algorithm within context. The mindfulness engendered by the algorithm then becomes embedded into the mental rules of thumb. We chose audit and feedback as part of our intervention, as it had been shown to be successful for reducing overuse of antibiotics in prior studies [12-17], and it is one of the evidence-based strategies recommended by guidelines on antimicrobial stewardship [18].

The local research assistant, supported by the Houston research coordinator, will gather episodes of positive urine cultures and classify the cases as ASB or UTI. The list of cases and their outcomes (ASB or UTI, treated appropriate or inappropriately) will be placed on each site's share drive on a weekly basis. The local pharmacy champion will deliver feedback on cases in real time (shortly after antibiotics are prescribed) through postprescription antimicrobial review, using the algorithm, and typically by telephone. The Houston-based team will also create appropriate teaching materials for each site's specific cases and place these on the shared drive, on a weekly basis. These case-based teaching materials will take the form of brief (10 min or less) PowerPoint presentations relevant to the cases that occurred in their site during the past week. The physician champion and research assistant will deliver audit and feedback via teaching cases in small group settings, such as resident teaching conferences, infectious diseases rounds with inpatient medicine teams, and hospitalist meetings. The goal is to deliver the audit and feedback to the relevant providers within a week of the positive urine culture report. The local champion will organize in services with 
the long-term care staff to present cases from long-term care. The research assistant will also receive pocket cards with the Kicking UTI algorithm for wide distribution within the local setting. Targets for audit and feedback delivery will be set, such as one case per week in acute care, two in-services per month in long-term care, and one teaching conference per quarter with residents and hospitalists.

\section{Economic evaluation}

The economic evaluation (Aim 3) will assess the financial implications of the intervention through a budget impact analysis (BIA). The BIA will be performed from the VHA's perspective using the best practice guidelines outlined by Sullivan et al. [30]. The BIA will estimate the financial impact per quarter on the VHA's budget to implement and sustain the intervention. The analysis will help the VHA and future VHA sites implementing the intervention to be financially prepared before initiating the intervention and will also establish the business case for the intervention. The intervention primarily aims to reduce clinical utilization as defined by the number of screening urine cultures ordered, unnecessary treatment of ASB, use of antimicrobials, and number of episodes of Clostridium difficile infection, without an increase in urinary source bacteremia. The BIA aims to capture the resource and cost changes associated with these reductions/changes, and also capture the cost of the intervention itself.

\section{Analysis}

\section{Analysis plan for aim 1}

Descriptive and summary statistics will be calculated using data from the surveys performed in Aim 1. For the Kicking CAUTI survey, we will calculate the knowledge score and will use identified gaps in knowledge to tailor audit and feedback materials. Guideline familiarity, social norms, outcome expectancy, self-reported behavior, and self-efficacy will be compared between sites and by characteristics such as type of facility. The other two surveys (ORCA and the site elements survey) will provide information relevant to local tailoring of the intervention.

\section{Analysis plan for Aim 2A}

The purpose of Aim 2 is to measure the effectiveness of the intervention in changing clinical outcomes of interest, in comparison to four control sites.

Variables for Aim 2A We will use data from the CDW to measure the effectiveness of the intervention in changing clinical outcomes of interest, in comparison to four control sites. The number of urine cultures at each site is the primary outcome, while secondary outcomes include the number of antibiotic courses started, number of antibiotic courses started within $48 \mathrm{~h}$ of a urine culture, and number of episodes of $C$. difficile infection. We will obtain the number of episodes of urinary source bacteremia (urosepsis) using a previously published definition (urine culture and blood culture have same organism and urine culture was collected within $0-7$ days prior to the blood culture) [31]. We will also obtain the number of patient days, or patient bed-days of care, to provide a denominator for standardization across facilities. The primary outcome is the total number of urine cultures ordered because ordering a urine culture is the first step that leads to overtreatment of ASB, and a positive urine culture is a powerful (but often incorrect) stimulus for use of antibiotics [32].

An additional set of measures will be obtained in both intervention and control sites by selecting patients with positive urine cultures from each site for chart review. Capturing whether a urinary catheter was present, whether each positive urine culture represented UTI, CAUTI or ASB, and whether antibiotics were given to treat the urine organisms will require chart review by a research assistant using our previously validated and standardized methods of chart review $[9,33]$. We expect high numbers of positive urine cultures from each site, and it will not be feasible or necessary to perform chart reviews for all positive cultures. We will randomly select patients with positive cultures from each site on a daily basis, varying the time of collection.

Analysis for Aim 2A We will use interrupted time series with segmented regression analysis (ITS/SRA) [34, 35]. Segmented regression analysis will be used to evaluate the effectiveness of the intervention in changing clinical outcomes of interest, in comparison to four control sites. We will conduct separate segmented regression analyses for the intervention and control sites. Data for each outcome variable will be aggregated monthly, and the time period will be divided into before, during, and after intervention segments, with separate intercepts and slopes estimated in each segment. Comparing the effect in the intervention sites with that in the control sites will allow separating the intervention effect from underlying secular trends $[34,36]$.

Power for Aim 2A Our study is powered around our primary outcome of interest, the number of urine cultures ordered (inappropriate screening for ASB). Based on our preliminary data, we expect intervention sites to provide approximately 900 urine cultures per month prior to the intervention, and 250 urine cultures per month after all sites are participating in the intervention. Because we will use existing data in CDW, we will be able to obtain our outcomes for 12 months pre- 
intervention (baseline), 12 months during the intervention, and 6-12 months post-intervention (sustainability phase) (Fig. 3). Our 36 monthly time points will provide adequate power to detect significant trends at different periods [37]. Segmented regression analysis will fit a model with three segments, corresponding to the pre-intervention, intervention, and post-intervention periods.

\section{Analysis plan for Aim 2B}

The purpose of Aim $2 \mathrm{~B}$ is to assess whether the adoption and fidelity (i.e. completeness of implementation) and the dose of the intervention are related to clinical outcomes (the number of urine cultures ordered).

Variables for Aim 2B The two variables of most interest used to measure "completeness" of implementation are the number of cases in which feedback was delivered out of the total number of cases of positive urine cultures and the number of participants in intervention activities out of eligible participants.

Analysis for Aim 2B The analysis for Aim 2B will assess whether more complete implementation is associated with better clinical outcomes (specifically the number of urine cultures ordered, standardized by bed-days). Data will be tabulated monthly for each VA facility.

Because facilities will have varying numbers of patients and urine cultures and because the outcome measures may have non-normal distributions, a preferred method of analysis involves the use of generalized linear mixed models (GLMM) or generalized estimating equations (GEE) [38]. An analysis such as GEE will allow monthly data to be nested within a facility. The regression models will include the month, facility, facility size, facility type, context (ORCA), patient characteristics, nurse staffing levels, antibiotic stewardship FTE, and the process measures representing the completeness of the implementation. To determine the subset of process measures most associated with the clinical outcome to use in the multivariable regression, we will initially conduct univariate analyses of each measure with the outcome. This includes correlation of continuous measures such as percentage of total cases per month that are presented as audit and feedback with the number of urine cultures ordered (standardized by bed-days). Analysis of variance will be used for assessing the association of categorical process measures, such as method of audit and feedback delivery (such as in person or by telephone), with the clinical outcome. Those process measures which are significant in the univariate analysis will be included in the GEE analysis. In the multivariable regression, the parameter estimates for process measures will indicate how much a change in the process measure will impact the clinical outcome. A negative parameter estimate will indicate that as the "completeness" of that implementation process measure increases, the clinical outcome (urine cultures) will decrease, after accounting for differences in variables such as facility size, ORCA, facility type (teaching or non-teaching), etc.

Power for Aim 2B We will have 12 monthly measurements of the clinical outcomes during the intervention year for each of the four facilities, hence a total of 48 measures. This sample size will allow the impact assessment of approximately five factors such as the facility type and the process measures on the clinical outcome, based on the general rule-of-thumb proposed by Cornfield of 10 observations for each independent variable in a linear regression model [39].

\section{Analysis plan for Aim 3}

The BIA will estimate costs for (1) the pre-intervention time period, in order to obtain the baseline utilization based on current practices, which contribute to clinical utilization costs of urine cultures ordered, use of antimicrobials, number of episodes of $C$. difficile infection, and urinary source bacteremia in the four VA medical centers before the intervention is put in place; (2) the intervention time period, in order to obtain clinical utilization costs during the intervention, in addition to the costs associated with the intervention implementation (including barrier assessment for initiating the intervention, intervention development, training and delivery); and (3) the post-intervention/sustainability time period, in order to capture the clinical utilization costs and other costs associated with sustaining the intervention i.e. training, delivery, and supervision involved with sustaining the intervention. Static modeling will be used for the BIA [40]. The patient population of interest is all inpatients on acute medical or long-term care wards at the intervention sites. The unit of analysis is a positive urine culture. The same patient may have more than one urine culture; we count these as separate episodes if more than 7 days apart. These recurrent episodes will be included in the BIA in order to capture the actual episode volume and costs for each VHA medical center. Inflation adjustment and discounting will not be applied because dollar values will be standardized to those from the final fiscal year of the study. Cost derived for the Kicking UTI intervention, Managerial Cost Accounting System (MCAS), and other clinical utilization costs will be adjusted for geographic variations. Cost derived from the standardized Health Economics Resource Center (HERC) data will not require geographic adjustments. 


\section{Ethical approval}

This study protocol has been approved by the institutional review board at Baylor College of Medicine and the Research \& Development Committee at the Michael E. DeBakey Veterans Affairs Medical Center, as the coordinating center. Each intervention site also obtained local IRB approval.

\section{Discussion}

The intervention we propose appears straightforward-convince providers to stop using antibiotics in asymptomatic patients when the antibiotics are unnecessary, potentially harmful, and costly. However, doing this practical work and doing it well requires changing physicians' and other health care providers' deeply held paradigms about the risk of bacteriuria (overestimated) and the risks of antibiotic use (underestimated) [41]. Historically, the healthy bladder was thought to be a sterile site, an assumption now proven incorrect by modern genomic sequencing, which demonstrates that the healthy bladder has robust bacterial and viral life within [42, 43]. Another barrier to the successful implementation of our stewardship intervention is the complexity of the practice guidelines themselves [44, 45] and the difficulty of applying 51 pages of evidence to an individual patient at the point of care. Additionally, the perceived needs of the individual patient in a moment of diagnostic uncertainty tend to outweigh concerns for theoretical future harms caused by damage to a patient's microbiome and selection for resistant organisms. The perceived needs of individual patients under care also tend to outweigh societal needs for effective antibiotics in the future.

We propose to counter these challenges with a solution that is relatively simple. We started with 51 pages of guidelines and created a multistep algorithm, with input from the guidelines' authors. We went through iterative revisions of the multistep algorithm with targeted endusers, and we distilled it into a two-step process that corrects cognitive biases and empowers the provider to withhold urine testing and treatment [9]. This algorithm is the focus of our audit and feedback activities. These simplifications were developed using the Evidence Integration Triangle framework, which emphasizes the need to keep interventions simple, participatory, and practical to promote rapid adoption of clinical practice guidelines [46].

Moving from our prior two-site study to a four-site intervention with four control sites might seem incremental, but this scale-up is an important shift in the translation pipeline. Our initial "Kicking CAUTI" study was in the T2 phase of knowledge dissemination-applying evidence based guidelines to improve clinical practice. Now with "Less is More" we move into T3 translation, because we are moving guidelines into widespread health practice through dissemination research $[47,48]$. We are also making a leap moving from a single, trusted local champion who originated the project to a more sophisticated model of internal and external facilitation. The original Kicking CAUTI campaign was successful in large part through the energy, involvement, and reputation of the trusted local leader. In order to disseminate beyond the reach of a single individual, yet maintain the role of the trusted local leader, we propose an internal/external facilitation model [21]. The Houston team will provide external facilitation in the form of subject matter expertise, teaching materials, and troubleshooting implementation barriers, while the local leaders, each well-known and charged with antibiotic stewardship in their home facility, will provide internal facilitation of the stewardship intervention [49].

Multiple organizational partners have an interest in helping our work succeed and planning for wide-scale dissemination. These partners, who will be involved in reviewing results and providing project direction on a twice yearly basis, include the VA National Center for Patient Safety, the VA Antimicrobial Stewardship Task Force, the VA National Infectious Diseases Service, and the Centers for Disease Control program "Get Smart for Healthcare." We also have a Veteran representative on the team so that we can have a bi-directional discussion with Veterans' associations about our work and how it can address Veterans' healthcare priorities.

In summary, "Less is More" moves from a proof-ofconcept effectiveness study to an implementation study involving significantly more sites, and uses the May's General Theory of Implementation to embed the intervention into normal processes of care with usual care providers. "Less is More" is the natural next step from our prior successful Kicking CAUTI intervention, with a more sophisticated approach to behavioral science and to evaluation of implementation.

\section{Acknowledgements \\ We have listed below all the co-authors who are members of the "Less is More" collaboration group. \\ The members of the Less is More study group include the following co-authors, in order: Barbara W. Trautner, Pooja Prasad, Larissa Grigoryan, Sylvia J. Hysong, Jennifer R. Kramer, Suja S. Rajan, Nancy J. Petersen, Tracey Rosen, Dimitri Drekonja, Christopher Graber, Payal Patel, Paola Lichtenberger, Timothy P. Gauthier, Steven Wiseman, Makoto Jones, Anne Sales, and Sarah Krein, and Aanand Naik.}

\section{Funding}

This work is supported by VA HSR\&D IIR 16-025 and by the Center for Innovations in Quality, Effectiveness and Safety (CIN 13-413) at the Michael E. DeBakey VA Medical Center, Houston, TX.

Availability of data and materials Not applicable. 


\section{Authors' contributions}

All authors contributed to the design of the study. PNP assisted with drafting and revising the manuscript. AES contributed the conceptual model. LG and NP designed the analytic plan, while SR created the budget impact analysis. BWT is the project lead. All authors have read and approved the final manuscript.

\section{Ethics approval and consent to participate}

Our study was approved by the Institutional Review Board of Baylor College of Medicine and the Research and Development Committee of the Michael E. DeBakey Veterans Affairs Medical Center.

\section{Consent for publication}

Not applicable.

\section{Competing interests}

The authors declare that they have no competing interests.

\section{Publisher's Note}

Springer Nature remains neutral with regard to jurisdictional claims in published maps and institutional affiliations.

\section{Author details}

${ }^{1}$ Center for Innovations in Quality, Effectiveness, and Safety (IQuESt) (152), Michael E. DeBakey Veterans Affairs Medical Center, 2002 Holcombe Boulevard, Houston, TX 77030, USA. ${ }^{2}$ Rice University, Houston, USA. ${ }^{3}$ Department of Family and Community Medicine, Baylor College of Medicine, 3701 Kirby Drive, Suite 600, Houston, TX 77098, USA. ${ }^{4}$ Department of Management, Policy and Community Heath, University of Texas (UT) School of Public Health (SPH), E-319, 1200 Pressler Street, Houston, TX 77030, USA. Infectious Diseases (111F), Minneapolis VA Medical Center, 1 Veterans Drive, Minneapolis, MN 55417, USA. ${ }^{6}$ Infectious Diseases Section, VA Greater Los Angeles Healthcare System, David Geffen School of Medicine at UCLA, 11301 Wilshire Blvd, 111-F, Los Angeles, CA 90073, USA. Division of Infectious Diseases, III-i, University of Michigan, 2215 Fuller Road, Ann Arbor, Ml 48105, USA. ${ }^{8}$ Bruce W. Carter VAMC, 1201 NW 16th Street, Miami, FL 33125-1693, USA. ${ }^{9}$ George E. Wahlen Veterans Affairs Medical Center, Mailstop 182, 500 Foothill Drive, Salt Lake City, UT 84148, USA. ${ }^{10}$ Department of Learning Health Sciences, University of Michigan Medical School, 209 Victor Vaughan Building, 2054, 1111 E. Catherine St, Ann Arbor, Ml 48109-2054, USA. ${ }^{11}$ VA Ann Arbor Center for Clinical Management Research, North Campus Research Complex, Building 16-333W, 2800 Plymouth Rd, Ann Arbor, MI 48109-2800, USA. ${ }^{12}$ Baylor College of Medicine in Houston, Houston, TX, USA.

\section{Received: 28 December 2017 Accepted: 9 January 2018}

\section{Published online: 19 January 2018}

\section{References}

1. Werner NL, Hecker MT, Sethi AK, Donskey CI. Unnecessary use of fluoroquinolone antibiotics in hospitalized patients. BMC Infect Dis. 2011;11(1):187.

2. Phillips CD, Adepoju O, Stone N, Moudouni DK, Nwaiwu O, Zhao H, Frentzel E, Mehr D, Garfinkel S. Asymptomatic bacteriuria, antibiotic use, and suspected urinary tract infections in four nursing homes. BMC Geriatr. 2012;12:73.

3. D'Agata E, Loeb MB, Mitchell SL. Challenges in assessing nursing home residents with advanced dementia for suspected urinary tract infections. J Am Geriatr Soc. 2013;61(1):62-6.

4. Gupta K, Hooton TM, Naber KG, Wullt B, Colgan R, Miller LG, Moran GJ, Nicolle LE, Raz R, Schaeffer AJ, et al. International clinical practice guidelines for the treatment of acute uncomplicated cystitis and pyelonephritis in women: a 2010 update by the infectious diseases society of America and the European society for microbiology and infectious diseases. Clin Infect Dis. 2011;52(5):e103-20.

5. Spivak ES, Burk M, Zhang R, Jones MM, Neuhauser MM, Goetz MB, Cunningham FE. Management of urinary tract infections medication use evaluation $\mathrm{g}$, management of urinary tract infections medication use evaluation g: management of bacteriuria in veterans affairs hospitals. Clin Infect Dis. 2017;65(6):910-7.

6. Trautner BW, Kelly PA, Petersen N, Hysong S, Kell H, Liao KS, Patterson JE, Naik AD. A hospital-site controlled intervention using audit and feedback to implement guidelines concerning inappropriate treatment of catheterassociated asymptomatic bacteriuria. Implement Sci. 2011;6(1):41.
7. Trautner BW, Grigoryan L, Petersen NJ, Hysong S, Cadena J, Patterson JE, Naik AD. Effectiveness of an antimicrobial stewardship approach for urinary catheterassociated asymptomatic Bacteriuria. JAMA Intern Med. 2015;175(7):1120-7.

8. Gigerenzer G, Gaissmaier W. Heuristic decision making. Annu Rev Psychol. 2011;62:451-82.

9. Trautner BW, Bhimani RD, Amspoker AB, Hysong SJ, Garza A, Kelly PA, Payne VL, Naik AD. Development and validation of an algorithm to recalibrate mental models and reduce diagnostic errors associated with catheterassociated bacteriuria. BMC Med Inform Decis Mak. 2013;13:48.

10. Flanders SA, Saint $S$. Enhancing the safety of hospitalized patients: who is minding the antimicrobials? Arch Intern Med. 2012;172(1):38-40.

11. Epstein RM. Mindful practice. JAMA. 1999;282(9):833-9.

12. Bonnal C, Baune B, Mion M, Armand-Lefevre L, L'Heriteau F, Wolmark Y, Lucet JC. Bacteriuria in a geriatric hospital: impact of an antibiotic improvement program. J Am Med Dir Assoc. 2008;9(8):605-9.

13. Linares $L A$, Thornton DJ, Strymish J, Baker E, Gupta K. Electronic memorandum decreases unnecessary antimicrobial use for asymptomatic bacteriuria and culture-negative pyuria. Infect Control Hosp Epidemiol. 2011;32(7):644-8.

14. Pettersson E, Vernby A, Molstad S, Lundborg CS. Can a multifaceted educational intervention targeting both nurses and physicians change the prescribing of antibiotics to nursing home residents? A cluster randomized controlled trial. J Antimicrob Chemother. 2011;66(11):2659-66.

15. Zabarsky TF, Sethi AK, Donskey CJ. Sustained reduction in inappropriate treatment of asymptomatic bacteriuria in a long-term care facility through an educational intervention. Am J Infect Control. 2008;36(7):476-80.

16. Cosgrove SE, Patel A, Song X, Miller RE, Speck K, Banowetz A, Hadler R, Sinkowitz-Cochran RL, Cardo DM, Srinivasan A. Impact of different methods of feedback to clinicians after postprescription antimicrobial review based on the centers for disease control and prevention's 12 steps to prevent antimicrobial resistance among hospitalized adults. Infect Control Hosp Epidemiol. 2007;28(6):641-6.

17. Cosgrove SE, Seo SK, Bolon MK, Sepkowitz KA, Climo MW, Diekema DJ, Speck K, Gunaseelan V, Noskin GA, Herwaldt LA, et al. Evaluation of postprescription review and feedback as a method of promoting rational antimicrobial use: a multicenter intervention. Infect Control Hosp Epidemiol. 2012;33(4):374-80.

18. Dellit TH, Owens RC, McGowan JE Jr, Gerding DN, Weinstein RA, Burke JP, Huskins WC, Paterson DL, Fishman NO, Carpenter CF, et al. Infectious diseases society of america and the society for healthcare epidemiology of America guidelines for developing an institutional program to enhance antimicrobial stewardship. Clin Infect Dis. 2007;44(2):159-77.

19. Barlam TF, Cosgrove SE, Abbo LM, MacDougall C, Schuetz AN, Septimus EJ, Srinivasan A, Dellit TH, Falck-Ytter YT, Fishman NO, et al. Implementing an antibiotic stewardship program: guidelines by the infectious diseases society of America and the society for healthcare epidemiology of America. Clin Infect Dis. 2016;62(10):e51-77.

20. May C. Towards a general theory of implementation. Implement Sci. 2013;8:18.

21. Stetler CB, Legro MW, Rycroft-Malone J, Bowman C, Curran G, Guihan M, Hagedorn $\mathrm{H}$, Pineros $\mathrm{S}$, Wallace CM. Role of "external facilitation" in implementation of research findings: a qualitative evaluation of facilitation experiences in the veterans health administration. Implement Sci. 2006;1:23.

22. Kirchner JE, Ritchie MJ, Pitcock JA, Parker LE, Curran GM, Fortney JC. Outcomes of a partnered facilitation strategy to implement primary caremental health. J Gen Intern Med. 2014;29(Suppl 4):904-12.

23. Mauskopf JA, Sullivan SD, Annemans L, Caro J, Mullins CD, Nuiiten M, Orlewska E, Watkins J, Trueman P. Principles of good practice for budget impact analysis: report of the ISPOR task force on good research practicesbudget impact analysis. Value Health. 2007;10(5):336-47.

24. May CR, Mair F, Finch T, MacFarlane A, Dowrick C, Treweek S, Rapley T, Ballini L, Ong BN, Rogers A, et al. Development of a theory of implementation and integration: normalization process theory. Implement Sci. 2009;4:29.

25. Saint $\mathrm{S}$, Gaies E, Fowler KE, Harrod M, Krein SL. Introducing a catheterassociated urinary tract infection (CAUTI) prevention guide to patient safety (GPS). Am J Infect Control. 2014;42(5):548-50.

26. Helfrich CD, Li YF, Sharp ND, Sales AE. Organizational readiness to change assessment (ORCA): development of an instrument based on the promoting action on research in health services (PARIHS) framework. Implement Sci. 2009:4:38.

27. Trautner BW, Petersen NJ, Hysong SJ, Horwitz D, Kelly PA, Naik AD. Overtreatment of asymptomatic bacteriuria: identifying provider barriers to evidence-based care. Am J Infect Control. 2014;42(6):653-8. 
28. Helfrich CD, Blevins D, Smith JL, Kelly PA, Hogan TP, Hagedorn H, Dubbert PM, Sales AE. Predicting implementation from organizational readiness for change: a study protocol. Implement Sci. 2011;6(1):76.

29. Hagedorn HJ, Heideman PW. The relationship between baseline organizational readiness to change assessment subscale scores and implementation of hepatitis prevention services in substance use disorders treatment clinics: a case study. Implement Sci. 2010;5:46.

30. Sullivan SD, Mauskopf JA, Augustovski F, Jaime Caro J, Lee KM, Minchin M, Orlewska E, Penna P, Rodriguez Barrios JM, Shau WY. Budget impact analysis-principles of good practice: report of the ISPOR 2012 budget impact analysis good practice II task force. Value Health. 2014;17(1):5-14.

31. Fortin E, Rocher I, Frenette C, Tremblay C, Quach C. Healthcare-associated bloodstream infections secondary to a urinary focus: the Quebec provincial surveillance results. Infect Control Hosp Epidemiol. 2012;33(5):456-62.

32. Leis JA, Rebick GW, Daneman N, Gold WL, Poutanen SM, Lo P, Larocque M, Shojania KG, McGeer A. Reducing antimicrobial therapy for asymptomatic bacteriuria among noncatheterized inpatients: a proof-of-concept study. Clin Infect Dis. 2014;58(7):980-3.

33. Burns AC, Petersen NJ, Garza A, Arya M, Patterson JE, Naik AD, Trautner BW. Accuracy of a urinary catheter surveillance protocol. Am J Infect Control. 2012;40(1):55-8.

34. Wagner AK, Soumerai SB, Zhang F, Ross-Degnan D. Segmented regression analysis of interrupted time series studies in medication use research. J Clin Pharm Ther. 2002;27(4):299-309.

35. Lagarde M. How to do (or not to do) ... Assessing the impact of a policy change with routine longitudinal data. Health Policy Plan. 2012;27(1):76-83.

36. Ramsay CR, Matowe L, Grilli R, Grimshaw JM, Thomas RE. Interrupted time series designs in health technology assessment: lessons from two systematic reviews of behavior change strategies. Int J Technol Assess Health Care. 2003;19(4):613-23.

37. Zhang F, Wagner AK, Ross-Degnan D. Simulation-based power calculation for designing interrupted time series analyses of health policy interventions. J Clin Epidemiol. 2011;64(11):1252-61.

38. Hussey MA, Hughes JP. Design and analysis of stepped wedge cluster randomized trials. Contemp Clin Trials. 2007;28(2):182-91.

39. Cornfield J. Recent methodological contributions to clinical trials. Am J Epidemiol. 1976;104(4):408-21.

40. Mauskopf JA, Earnshaw S, Mullins CD. Budget impact analysis: review of the state of the art. Expert Rev Pharmacoecon Outcomes Res. 2005;5(1):65-79.

41. Brehaut JC, Poses R, Shojania KG, Lott A, Man-Son-Hing M, Bassin E, Grimshaw J. Do physician outcome judgments and judgment biases contribute to inappropriate use of treatments? Study protocol. Implement Sci. 2007:2:18.

42. Malki K, Sible E, Cooper A, Garretto A, Bruder K, Watkins SC, Putonti C. Seven Bacteriophages Isolated from the Female Urinary Microbiota. Genome Announc. 2016:4(6):e01003-16.

43. Wolfe AJ, Brubaker L. "sterile urine" and the presence of bacteria. Eur Urol. 2015;68(2):173-4.

44. Nicolle LE, Bradley S, Colgan R, Rice JC, Schaeffer A, Hooton TM. Infectious diseases society of America guidelines for the diagnosis and treatment of asymptomatic bacteriuria in adults. Clin Infect Dis. 2005;40(5):643-54

45. Hooton TM, Bradley SF, Cardenas DD, Colgan R, Geerlings SE, Rice JC, Saint S, Schaeffer AJ, Tambayh PA, Tenke P, et al. Diagnosis, prevention, and treatment of catheter-associated urinary tract infection in adults: 2009 international clinical practice guidelines from the infectious diseases society of America. Clin Infect Dis. 2010;50(5):625-63.

46. Naik AD, Skelton F, Amspoker AB, Glasgow RA, Trautner BW. A fast and frugal algorithm to strengthen diagnosis and treatment decisions for catheter-associated bacteriuria. PLoS One. 2017;12(3):e0174415.

47. Milat AJ, Li B. Narrative review of frameworks for translating research evidence into policy and practice. Public Health Res Pract. 2017;27(1):e2711704.

48. Khoury MJ, Gwinn M, loannidis JP. The emergence of translational epidemiology: from scientific discovery to population health impact. Am J Epidemiol. 2010;172(5):517-24.

49. Ritchie MJ, Dollar KM, Miller CJ, Oliver KA, Smith JL, Lindsay JA, Kirchner JE: Using Implementation Facilitation to Improve Care in the Veterans Health Administration (Version 2). In: Veterans Health Administration, Quality Enhancement Research Initiative (QUERI) for Team-Based Behavioral Health. 2017. https://www.queri.research.va.gov/tools/implementation/Facilitation-Manual.pdf.

\section{Submit your next manuscript to BioMed Central and we will help you at every step:}

- We accept pre-submission inquiries

- Our selector tool helps you to find the most relevant journal

- We provide round the clock customer support

- Convenient online submission

- Thorough peer review

- Inclusion in PubMed and all major indexing services

- Maximum visibility for your research

Submit your manuscript at www.biomedcentral.com/submit
Ciomed Central 